\title{
Pyridine Based Chiral Smectogens: Effects of Polar End Groups on Liquid Crystal Properties
}

Deniz $\operatorname{Vardar}^{1}$, Huriye Akdaş-Kılıç ${ }^{1,2}$, Hale Ocak ${ }^{1 *}$, Olivier Jeannin ${ }^{2}$, Franck Camerel$^{2}$, Belkız

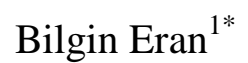

${ }^{1}$ Yildiz Technical University, Department of Chemistry, 34220 Esenler, Istanbul, Turkey

${ }^{2}$ Univ Rennes, CNRS, ISCR - UMR 6226, F-35000 Rennes

*Corresponding Author: bbilgin@yildiz.edu.tr, hocak@yildiz.edu.tr

\begin{abstract}
:
In this study, novel chiral pyridine-based rod-like mesogens, consisting of a chloro or bromo group substituted pyridine head core which is connected to one or two aromatic rings through ester linkers and a flexible $(S)$-2-methylbutoxy or (S)-3,7-dimethyloctyloxy chiral end chain have been synthesized. The liquid crystal properties of new compounds were investigated by various experimental techniques such as DSC, POM and SAXS. Depending of the nature of the halogen end group, the derivatives with two aromatic rings are non-mesogenic compounds whereas a smectic A phase (SmA) enantiotropically occurs for the members with three aromatic rings of the series. Additionally, chloro group substituted compound composed of three aromatic rings carrying a $(S)$-2-methylbutoxy end chain exhibits an enantiotropic chiral nematic phase $\left(\mathrm{N}^{*}\right)$.
\end{abstract}

Keywords: Liquid crystal, rod-like molecules, chirality, pyridine-based cores. 


\section{Introduction}

Liquid crystals are advanced organic materials which have found a wide usage in indicator technology, organic semiconductor, sensors, bioactive materials and optical device applications.[1,2,3,4] Studies of the structure-mesogenity relationship in liquid crystals have led to significant improvements in the design of mesogenic molecules.[5,6] Molecular design plays an important role in the emergence of liquid crystalline properties and a rational design based on established molecular guidelines can allow for a rapid optimization of the liquid crystalline properties, in term of structural organization and thermal range, for the target application.[7,8] Within the different classes of LCs, rod-like (calamitic) liquid crystals, characterized by their simple molecular structure and sensitivity to external stimulating effects such as electric and magnetic fields, are intensively studied and incorporated in many LC materials. $[9,10,11]$

The calamitic structures usually consist of central rigid core, which ensure chemical anisotropy to the structure, with flexible chains at the end positions. Their liquid crystal properties can be tuned by varying the aromatic core, the type and length of end chains and incorporating of branched units or molecular chirality.[12,13] In the design of a calamitic liquid crystal molecule, the number of aromatic rings that determine the length of the molecule, the type of binding units, the presence of polar substituents on the aromatic core, and the type of the flexible aliphatic ends chains are the major factors that determine the emergence of mesomorphic properties. The most commonly used terminal groups in calamitic molecules are long alkyl and alkyloxy chains. By providing flexibility feature to the rigid core unit, they also play an important role in decreasing the melting point of the molecule and also contribute to mesophase formation. In addition, any polar end group (cyano or halogen, etc.) in the structure, helps the formation of a more regular mesophase because it has high polarity and can contribute to molecular stacking, which can positively influence the mesophase formation.[14]

The introduction of a chiral center placed on the central core, on the terminal chains or on the flexible linking units can lead to liquid crystals which exhibit different properties than structurally related to those of non-chiral analogous.[15,16,17,18,19] A helical arrangement of the molecules can emerge, leading to a polar organization of the chiral molecules, which is one of the main ways to obtain light-responsive liquid crystal materials for applications in high-resolution displays and electro-optical devices.[7,20,21] Besides, helical LC phases can also arise from achiral molecules with a bent geometry.[22,23,24] 
The introduction of a heteroatom in the molecular structure are decisive factors for the formation in a particular type of molecular organization at a specific temperature range. In particular, the number and the position into the aromatic ring affects its polarizability and sometimes the geometric shape of the molecule, and as a result, the physical properties of the mesogen such as the phase transition temperatures and/or the dielectric properties.[25] Sixmembered N-heterocyclic systems such as pyridine is one of the simplest heterocyclic aromatics used as a core system in the design of liquid crystalline molecules.[26,27,28,29,30] The position of the nitrogen atom in the pyridine fragment and its position in the molecular core considerably influence the liquid crystalline properties of pyridine derivatives[31] and has a significant impact on the dispersion forces, the dipole moment, the polarizability of the molecule and, inevitably, on the dielectric nature of the mesogen.[32]

Herein we report the design, synthesis and characterization of new pyridine-based calamitic mesogens, consisting of a chloro or bromo group substituted pyridine head core which is connected to one or two aromatic rings through ester linkers and carrying a $(S)$-2methylbutoxy or a (S)-3,7-dimethyloctyloxy chiral side chain. The effect of structural variations on mesomorphic properties have been discussed based on some viewpoints: the introduction of two chiral chains with different length, the number of aromatic rings in the rigid core unit, the attaching of a pyridine ring into the core unit and the presence of polar end groups such as bromo and chloro. Their thermal and liquid crystal properties were investigated by differential scanning calorimetry (DSC), optical polarizing microscopy (POM) and Small-angle X-ray scattering (SAXS).

\section{Experimental}

\subsection{Materials and instrumentation}

New chiral compounds (4a,b, 7a,b and $\mathbf{8 a , b})$ were characterized by ${ }^{1} \mathrm{H}$ NMR and ${ }^{13} \mathrm{C}$ NMR (Bruker Avance III 500 spectrometer, in $\mathrm{CDCl}_{3}$ solution, with tetramethylsilane as external standard). Microanalysis was performed using a Thermo Fischer Scientific FlashEA 1112 Series elemental analyzer.

The mesomorphic properties of compounds were investigated by using a Mettler FP-82 HT hot stage and control unit in conjunction with a Leica DM2700P polarizing microscope. DSCthermograms of chiral imine compounds were recorded on a Perkin-Elmer DSC-6 with heating and cooling rate of $10{ }^{\circ} \mathrm{C} \cdot \mathrm{min}^{-1}$ under nitrogen atmosphere. 
X-ray scattering experiments (SAXS) were performed using a Microstar Bruker rotating anode $\mathrm{X}$-ray generator operated at $40 \mathrm{kV}$ and $40 \mathrm{~mA}$ with monochromatic $\mathrm{Cu} \mathrm{K} \alpha$ radiation $(\lambda$ $=1.541 \AA$ ) and point collimation. The patterns were collected with a Mar345 Image-Plate detector (Marresearch, Norderstedt, Germany). The exposure time at each temperature was $3600 \mathrm{~s}$ and the heating or cooling speed between two temperatures was $10{ }^{\circ} \mathrm{C} \cdot \mathrm{min}^{-1}$. The samples were held in Lindeman glass capillaries (1.5 $\mathrm{mm}$ diameter). The capillaries were placed inside a Linkam HFX350-Capillary X-Ray stage which allow measurements from -196 ${ }^{\circ} \mathrm{C}$ up to $350{ }^{\circ} \mathrm{C}$ with an accuracy of $0.1{ }^{\circ} \mathrm{C}$.

$(S)-(-)-\beta$-Citronellol (Aldrich, $\geq 99.0 \%,[\alpha] \frac{20}{589}=-5.3^{\circ}$, neat), (S)-(-)-2-methyl-1-butanol (Fluka), p-toluenesulfonyl chloride (Merck), 4-benzyloxyphenol (Alfa Aesar), ethyl 4hydroxybenzoate (Alfa Aesar), benzyl chloride (Merck), $\mathrm{K}_{2} \mathrm{CO}_{3}$ (Merck), sodium hydroxide pellets (Merck), N,N'-dicyclohexylcarbodiimide (Merck), 4-(dimethylamino)pyridine (Merck), 10\% Pd/C (Alfa Aesar), methyl 5-bromopyridine-2-carboxylate (ABCR), methyl 6chloropyridine-3-carboxylate (Aldrich), were purchased from commercial sources and used as received. Ethanol (Merck), 2-butanone (Merck) and THF (Merck \%99) were purchased commercially as dry solvents and were used without further purification. Anhydrous solvent $\mathrm{CH}_{2} \mathrm{Cl}_{2}$ was dried over di-phosphorus pentoxide (Merck) and distilled under a $\mathrm{N}_{2}$ atmosphere. Solvents used in the purification step such as crystallization and column chromatography (hexane $(\mathrm{H})$, ethyl acetate (EA), chloroform, dichloromethane and ethanol) were distilled. Analytical thin-layer chromatography (TLC) was carried out on aluminium plates coated with silica gel 60 F254 (Merck) for intermediates and aluminium plates coated with aluminium oxide 60 F254, neutral (Merck) for target imine compounds. Column chromatography was performed using silica gel 60 (Merck, pore size $60 \AA$, 230-400 mesh particle size).

\subsection{Synthesis and Characterization}

The synthesis of new pyridine based chiral calamitic compounds $\mathbf{4 a , b}$ and compounds $\mathbf{7 a}, \mathbf{b}$ and $\mathbf{8 a}, \mathbf{b}$ are presented in Scheme 1. 


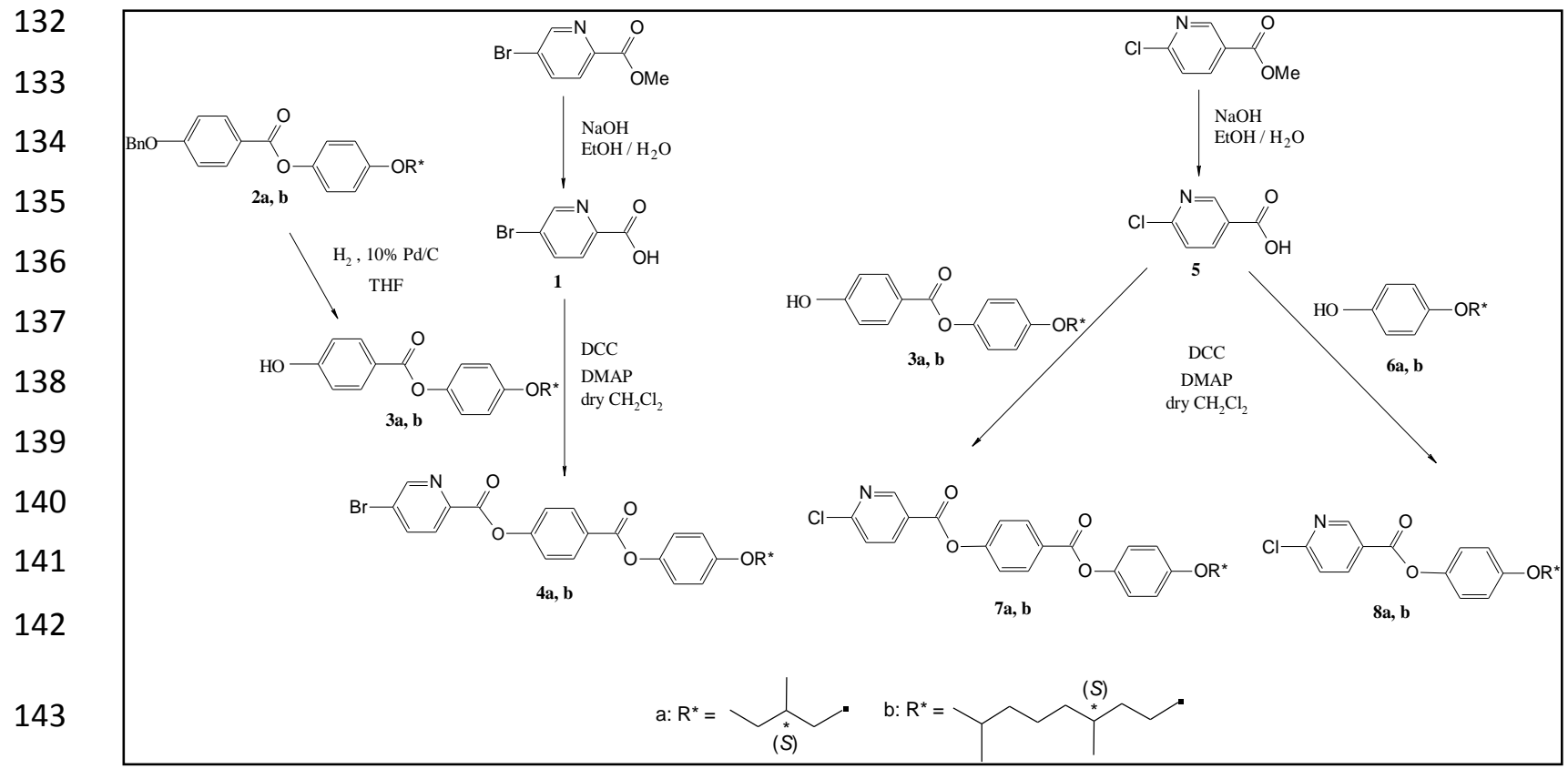

Scheme 1. Synthesis of the new pyridine based chiral calamitic compounds $4 \mathbf{a}, \mathbf{b}, \mathbf{7 a}, \mathbf{b}$ and 8a,b.

New pyridine based chiral compounds $\mathbf{4 a}, \mathbf{b}$ and $\mathbf{7 a}, \mathbf{b}$ with three aromatic cores, in which the pyridine ring substituted with a bromo or chloro polar end group have been prepared by the esterification of 4-Alkyloxyphenyl-4-hydroxybenzoates (3a, b) and 5-bromopyridine-2carboxylic acid (1) or 6-chloropyridine-3-carboxylic acid (5), which were obtained basic hydrolysis in EtOH with $\mathrm{NaOH}(\mathrm{aq})$, using N,N'-dicyclohexylcarbodiimide (DCC) and 4(dimethylamino)pyridine (DMAP) as catalyst in dry $\mathrm{CH}_{2} \mathrm{Cl}_{2}$. As described previously[33],

Dimethyloctyloxy)phenyl 4-hydroxybenzoate (3b)[33], have been synthesized starting from (S)-2-methylbutanol, which was tosylated to yield (S)-2-methylbutyl-1-tosylate[13] and (S)-()- $\beta$-citronellol, which was firstly hydrogenated $\left(\mathrm{H}_{2}, \mathrm{Pd} / \mathrm{C}\right.$ in $\left.\mathrm{MeOH}\right)$ to give $(S)$-3,7-dimethyl1-octanol and then converted to (S)-3,7-dimethyloctyl-1-bromide (conc. aqu. $\mathrm{HBr}$, conc. $\left.\mathrm{H}_{2} \mathrm{SO}_{4}\right)$ [34], respectively. The etherification of (S)-2-methylbutyl-1-tosylate or (S)-3,7dimethyloctyl-1-bromide with 4-benzyloxyphenol, followed by hydrogenolytic debenzylation yielded to the corresponding 4-alkyloxyphenols $\mathbf{6 a}$ and $\mathbf{6 b}$.[35,36] The esterification of the obtained 4-alkyloxyphenols with 4-benzyloxybenzoic acid[37] using $N, N^{\prime}$ dicyclohexylcarbodiimide (DCC) and 4-(dimethylamino)pyridine (DMAP) yielded (S)-4-(2methylbutoxy)phenyl 4-benzyloxybenzoate (2a) or (S)-4-(3,7-Dimethyloctyloxy)phenyl 4- 
benzyloxybenzoate (2b) and followed by deprotection of the benzyl group under hydrogenolytic reaction conditions leads to 4-Alkyloxyphenyl-4-hydroxybenzoates (3a, b). The esterification of 6-chloropyridine-3-carboxylic acid (5) with the corresponding 4alkyloxyphenol 6a,b using the same procedure yielded to 4-((S)-2-methylbutoxy)phenyl 6chloronicotinate (8a) and 4-((S)-3,7-dimethyloctyloxy)phenyl 6-chloronicotinate respectively.

Spectroscopic data and preparation procedures of (S)-4-(3,7-Dimethyloctyloxy)phenyl 4benzyloxybenzoate (2b), (S)-4-(3,7-Dimethyloctyloxy)phenyl 4-hydroxybenzoate (3b) and (S)-4-(3,7-Dimethyloctyloxy)phenol (6b) were given in Guzeller et al.[33] The procedure for the synthesis of $\mathbf{3 a}$ and spectroscopic data of $\mathbf{2 a}, \mathbf{3 a}, \mathbf{6 a}$ as well as 5-bromopyridine-2carboxylic acid (1) or 6-chloropyridine-3-carboxylic acid (5) were given in supplementary information (SI) file.

\section{The procedure for the synthesis of the final compounds $4 a, b, 7 a, b$ and $8 a, b$ :}

For the synthesis of $\mathbf{4 a}, \mathbf{b}$ and $\mathbf{7 a}, \mathbf{b}$, to a mixture of $1.5 \mathrm{mmol}$ of 5-bromopyridine-2-carboxylic acid (1) or 6-chloropyridine-3-carboxylic acid (5) and $1.5 \mathrm{mmol}$ of (S)-4-(2methylbutoxy)phenyl 4-hydroxybenzoate (3a) or (S)-4-(3,7-Dimethyloctyloxy)phenyl 4hydroxybenzoate (3b)[33] in $20 \mathrm{~mL}$ of dichloromethane, $N, N$ '-dicyclohexylcarbodiimide $(2.5$ mmol) and 4-(dimethylamino)pyridine $(0.12 \mathrm{mmol})$ were added. The mixture was stirred under argon atmosphere at room temperature for 24 hours and the reaction progress was monitored by TLC (H: EA / 3: 1). The reaction mixture was filtered over silica gel and the solvent was removed under reduced pressure. The crude product was purified by column chromatography on silica gel eluting with hexane/ethylacetate mixtures (H: EA / 3: 1) and finally washed with ethanol.

The synthesis of compounds $\mathbf{8 a , b}$ has been carried out by the esterification of 6chloropyridine-3-carboxylic acid (5) and the corresponding 4-alkyloxyphenols $\mathbf{6 a}$ and $\mathbf{6 b}$ [33] using the same procedure in that of target products $\mathbf{4 a , b}$ and $\mathbf{7 a , b}$.

\section{4-[4-((S)-2-Methylbutoxy)phenoxycarbonyl]phenyl 5-bromopicolinate (4a)}

Yield: $35 \%$; colorless crystals. ${ }^{1} \mathbf{H}$ NMR $\left(500 \mathrm{MHz} \mathrm{CDCl}_{3}\right): \delta(\mathrm{ppm})=8.84(\mathrm{~d}, J \approx 1.9 \mathrm{~Hz}$, $1 \mathrm{H}, \mathrm{Ar}-\mathrm{CH}), 8.23(\mathrm{~d}, J \approx 8.7 \mathrm{~Hz}, 2 \mathrm{H}, \mathrm{Ar}-\mathrm{CH}), 8.12(\mathrm{~d}, J \approx 8.3 \mathrm{~Hz}, 1 \mathrm{H}, \mathrm{Ar}-\mathrm{CH}), 8.02\left(\mathrm{dd}, J_{1} \approx\right.$ $\left.8.3, J_{2} \approx 2.3 \mathrm{~Hz}, 1 \mathrm{H}, \mathrm{Ar}-\mathrm{CH}\right), 7.34(\mathrm{~d}, J \approx 8.7 \mathrm{~Hz}, 2 \mathrm{H}, \mathrm{Ar}-\mathrm{CH}), 7.04(\mathrm{~d}, J \approx 9.0 \mathrm{~Hz}, 2 \mathrm{H}, \mathrm{Ar}-$ $\mathrm{CH}), 6.87(\mathrm{~d}, J \approx 9.0 \mathrm{~Hz}, 2 \mathrm{H} \mathrm{Ar}-\mathrm{CH}), 3.76,3.67\left(2 \mathrm{dd}, J_{1} \approx 8.9, J_{2} \approx 6.0 \mathrm{~Hz}, 2 \mathrm{H}, \mathrm{OCH}_{2}\right), 1.81-$ 
198

199

200

201

202

203

204

205

206

207

208

209

210

211

212

213

214

215

216

217

218

219

220

221

222

223

224

225

226

227

228

229

230

$1.77(\mathrm{~m}, 1 \mathrm{H}, \mathrm{CH}), 1.24-1.16\left(\mathrm{~m}, 2 \mathrm{H}, \mathrm{CH}_{2}\right), 0.95\left(\mathrm{~d}, J \approx 6.7 \mathrm{~Hz}, 3 \mathrm{H}, \mathrm{CH}_{3}\right), 0.89(\mathrm{t}, J \approx 7.5 \mathrm{~Hz}$, $\left.3 \mathrm{H}, \mathrm{CH}_{3}\right) .{ }^{13} \mathrm{C}$ NMR $\left(125 \mathrm{MHz}, \mathrm{CDCl}_{3}\right): \delta(\mathrm{ppm})=164.70,162.78(\mathrm{COO}), 157.16,154.72$, 145.35, 144.07, 127.77, 126.01 (Ar-C), 151.50, 140.02, 131.90, 127.09, 122.30, 121.89, $115.14(\mathrm{Ar}-\mathrm{CH}), 73.29\left(\mathrm{OCH}_{2}\right), 34.70(\mathrm{CH}), 26.12\left(\mathrm{CH}_{2}\right), 16.52,11.31\left(\mathrm{CH}_{3}\right)$. FT-IR: $\gamma\left(\mathrm{cm}^{-}\right.$ $\left.{ }^{1}\right)=1758,1735$ ( $\mathrm{C}=\mathrm{O}$ stretching), 1603-1505 (Ar-C=C stretching). $\mathbf{C}_{\mathbf{2 4}} \mathbf{H}_{22} \mathbf{B r N O}_{\mathbf{5}}$ (484.35); Anal. Calc. (\%): C, 59.52; H, 4.58; N, 2.89. Found (\%): C, 59.23; H, 4.91; N, 3.03.

\section{4-[4-((S)-3,7-Dimethyloctyloxy)phenoxycarbonyl]phenyl 5-bromopicolinate (4b)}

Yield: $34 \%$; colorless crystals. ${ }^{1} \mathbf{H}$ NMR $\left(500 \mathrm{MHz} \mathrm{CDCl}_{3}\right): \delta(\mathrm{ppm})=8.84(\mathrm{~d}, J \approx 1.8 \mathrm{~Hz}$, $1 \mathrm{H}, \mathrm{Ar}-\mathrm{CH}), 8.23(\mathrm{~d}, J \approx 8.8 \mathrm{~Hz}, 2 \mathrm{H}, \mathrm{Ar}-\mathrm{CH}), 8.12(\mathrm{~d}, J \approx 8.6 \mathrm{~Hz}, 1 \mathrm{H}, \mathrm{Ar}-\mathrm{CH}), 8.02\left(\mathrm{dd}, J_{l} \approx\right.$ 8.3, $\left.J_{2} \approx 2.3 \mathrm{~Hz}, 1 \mathrm{H}, \mathrm{Ar}-\mathrm{CH}\right), 7.34(\mathrm{~d}, J \approx 8.8 \mathrm{~Hz}, 2 \mathrm{H}, \mathrm{Ar}-\mathrm{CH}), 7.05$ (d, $J \approx 9.0 \mathrm{~Hz}, 2 \mathrm{H}, \mathrm{Ar}-$ $\mathrm{CH}), 6.87(\mathrm{~d}, J \approx 9.0 \mathrm{~Hz}, 2 \mathrm{H}, \mathrm{Ar}-\mathrm{CH}), 3.98-3.86\left(\mathrm{~m}, 2 \mathrm{H}, \mathrm{OCH}_{2}\right), 1.81-1.70(\mathrm{~m}, 1 \mathrm{H}, \mathrm{CH})$, 1.57-1.08 (m, 9H, CH, CH$), 0.88\left(\mathrm{~d}, J \approx 6.6 \mathrm{~Hz}, 3 \mathrm{H}, \mathrm{CH}_{3}\right), 0.81\left(\mathrm{~d}, J \approx 6.6 \mathrm{~Hz} ; 6 \mathrm{H}, \mathrm{CH}_{3}\right)$.

${ }^{13}$ C NMR $\left(125 \mathrm{MHz}, \mathrm{CDCl}_{3}\right): \delta(\mathrm{ppm})=164.69,162.79$ (COO), 156.97, 154.72, 145.34, 144.10, 127.77, 126.01 (Ar-C), 151.50, 140.03, 131.90, 127.09, 122.32, 121.90, 115.13 (Ar$\mathrm{CH}), 66.74\left(\mathrm{OCH}_{2}\right), 39.24$, 37.27, 36.20, $24.66\left(\mathrm{CH}_{2}\right), 29.83,27.97(\mathrm{CH}), 22.71,22.61,19.64$ $\left(\mathrm{CH}_{3}\right)$. FT-IR: $\gamma\left(\mathrm{cm}^{-1}\right)=1734(2 \mathrm{C}=\mathrm{O}$ stretching), 1593-1506 (Ar-C=C stretching). $\mathbf{C}_{29} \mathrm{H}_{32} \mathrm{BrNO}_{5}$ (554.48); Anal. Calc. (\%): C, 62.82; H, 5.82; N, 2.53. Found (\%): C, 63.13; H, $5.90 ; \mathrm{N}, 2.21$.

\section{4-[4-((S)-2-Methylbutoxy)phenoxycarbonyl]phenyl 6-chloronicotinate (7a)}

Yield: $45 \%$; colorless crystals. ${ }^{1} \mathbf{H}$ NMR $\left(500 \mathrm{MHz} \mathrm{CDCl}_{3}\right): \delta(\mathrm{ppm})=9.20(\mathrm{~d}, J \approx 2.4 \mathrm{~Hz}$, $1 \mathrm{H}, \mathrm{Ar}-\mathrm{CH}), 8.42\left(\mathrm{dd}, J_{l} \approx 8.3 \mathrm{~Hz}, J_{2} \approx 2.4 \mathrm{~Hz}, 1 \mathrm{H}, \mathrm{Ar}-\mathrm{CH}\right), 8.31(\mathrm{~d}, J \approx 8.8 \mathrm{~Hz}, 2 \mathrm{H}, \mathrm{Ar}-\mathrm{CH})$, $7.53(\mathrm{~d}, J \approx 8.3 \mathrm{~Hz}, 1 \mathrm{H}, \mathrm{Ar}-\mathrm{CH}), 7.39$ (d, $J \approx 8.8 \mathrm{~Hz}, 2 \mathrm{H}, \mathrm{Ar}-\mathrm{CH}), 7.12(\mathrm{~d}, J \approx 9.0 \mathrm{~Hz}, 2 \mathrm{H}, \mathrm{Ar}-$ $\mathrm{CH}), 6.95(\mathrm{~d}, J \approx 9.0 \mathrm{~Hz}, 2 \mathrm{H}, \mathrm{Ar}-\mathrm{CH}), 3.83,3.75\left(2 \mathrm{dd}, J_{1} \approx 8.9 \mathrm{~Hz}, J_{2} \approx 6.0 \mathrm{~Hz}, 2 \mathrm{H}, \mathrm{OCH}_{2}\right)$, 1.93-1.82 (m, $1 \mathrm{H}, \mathrm{CH}), 1.66-1.50,1.35-1.23\left(2 \mathrm{~m}, 2 \mathrm{H}, \mathrm{CH}_{2}\right), 1.03\left(\mathrm{~d}, J \approx 6.7 \mathrm{~Hz}, 3 \mathrm{H}, \mathrm{CH}_{3}\right)$, $0.96\left(\mathrm{t}, J \approx 7.5 \mathrm{~Hz}, 3 \mathrm{H}, \mathrm{CH}_{3}\right) .{ }^{13} \mathbf{C} \mathbf{N M R}\left(125 \mathrm{MHz}, \mathrm{CDCl}_{3}\right): \delta(\mathrm{ppm})=164.64,162.54$ (COO), 157.19, 156.72, 154.27, 144.04, 127.85, 124.13 (Ar-C), 151.72, 140.09, 131.94, 124.54, 122.29, 121.76, $115.15(\mathrm{Ar}-\mathrm{CH}), 73.30\left(\mathrm{OCH}_{2}\right), 34.71(\mathrm{CH}), 26.12\left(\mathrm{CH}_{2}\right), 16.52$, $11.31\left(\mathrm{CH}_{3}\right)$. FT-IR: $\gamma\left(\mathrm{cm}^{-1}\right)=1738,1712(\mathrm{C}=\mathrm{O}$ stretching $), 1605-1507$ (Ar-C=C stretching). $\mathbf{C}_{\mathbf{2 4}} \mathbf{H}_{22} \mathbf{C I N O}_{5}$ (439.89); Anal. Calc. (\%): C, 65.53; H, 5.04; N, 3.18. Found (\%): C, 65.66; H, 5.16; N, 2.97. Full MS (ESI) (electrospray ionization) (+): m/z (\%) = 440 (100) $\left[\mathrm{M}^{+}\right], 260$ (87) $\left[\mathrm{M}^{+}-\mathrm{C}_{11} \mathrm{H}_{15} \mathrm{O}_{2}\right], 140$ (25) $\left[\mathrm{C}_{6} \mathrm{H}_{3} \mathrm{ClNO}\right]$. 
Yield: $45 \%$; colorless crystals. ${ }^{1} \mathbf{H}$ NMR $\left(500 \mathrm{MHz}, \mathrm{CDCl}_{3}\right): \delta(\mathrm{ppm})=9.20(\mathrm{~d}, J \approx 2.4,1 \mathrm{H}$, $\operatorname{Ar}-\mathrm{C} H), 8.42\left(\mathrm{dd}, J_{1} \approx 8.3 \mathrm{~Hz}, J_{2} \approx 2.4 \mathrm{~Hz}, 1 \mathrm{H}, \mathrm{Ar}-\mathrm{C} H\right), 8.31(\mathrm{~d}, J \approx 8.8 \mathrm{~Hz}, 2 \mathrm{H}, \mathrm{Ar}-\mathrm{CH})$, $7.53(\mathrm{~d}, J \approx 8.3,1 \mathrm{H}, \mathrm{Ar}-\mathrm{CH}), 7.39(\mathrm{~d}, J \approx 8.8 \mathrm{~Hz}, 2 \mathrm{H}, \mathrm{Ar}-\mathrm{CH}), 7.13(\mathrm{~d}, J \approx 9.0 \mathrm{~Hz}, 2 \mathrm{H}, \mathrm{Ar}-$ $\mathrm{CH}), 6.95(\mathrm{~d}, J \approx 9.0 \mathrm{~Hz}, 2 \mathrm{H}, \mathrm{Ar}-\mathrm{CH}), 4.09-3.89\left(\mathrm{~m}, 2 \mathrm{H}, \mathrm{OCH}_{2}\right), 1.88-1.78(\mathrm{~m}, 1 \mathrm{H}, \mathrm{CH})$, 1.65-1.13 (m, 9H, CH, $4 \mathrm{CH}_{2}$ ), 0.95 (d, $\left.J \approx 6.6 \mathrm{~Hz}, 3 \mathrm{H}, \mathrm{CH}_{3}\right), 0.88\left(\mathrm{~d}, J \approx 6.1 \mathrm{~Hz}, 6 \mathrm{H}, \mathrm{CH}_{3}\right)$. ${ }^{13}$ C NMR $\left(125 \mathrm{MHz}, \mathrm{CDCl}_{3}\right): \delta(\mathrm{ppm})=164.64,162.55$ (COO), 156.99, 156.67, 154.26, 144.05, 127.84, 124.12 (Ar-C), 151.73, 140.10, 131.95, 124.55, 122.31, 121.77, 115.13 (Ar$\mathrm{CH}), 66.73\left(\mathrm{OCH}_{2}\right)$, 39.24, 37.27, 36.19, $24.66\left(\mathrm{CH}_{2}\right)$, 29.82, $27.98(\mathrm{CH})$, 22.72, 22.64, 19.64 $\left(\mathrm{CH}_{3}\right)$. FT-IR: $\gamma\left(\mathrm{cm}^{-1}\right)=1736,1724(\mathrm{C}=\mathrm{O}$ stretching), 1605-1507 (Ar-C=C stretching). $\mathbf{C}_{29} \mathrm{H}_{32} \mathrm{CINO}_{5}$ (510.03); Anal. Calc. (\%): C, 68.29; H, 6.32; N, 2.75. Found (\%): C, 68.00; H, 6.30; N, 2.70. Full MS (ESI) (electrospray ionization) $(+): \mathrm{m} / \mathrm{z}(\%)=512(18)\left[\mathrm{M}^{+}+2 \mathrm{H}\right], 260$ (100) $\left[\mathrm{M}^{+}-\mathrm{C}_{16} \mathrm{H}_{25} \mathrm{O}_{2}\right], 140(65)\left[\mathrm{C}_{6} \mathrm{H}_{3} \mathrm{ClNO}\right]$.

\section{4-((S)-2-Methylbutoxy)phenyl 6-chloronicotinate (8a)}

Yield: $48 \%$; colorless crystals. ${ }^{\mathbf{1}} \mathbf{H}$ NMR $\left(500 \mathrm{MHz}_{\mathrm{CDCl}}\right): \delta(\mathrm{ppm})=9.09(\mathrm{~d}, J \approx 2.4 \mathrm{~Hz}$, $1 \mathrm{H}, \mathrm{Ar}-\mathrm{CH}), 8.31\left(\mathrm{dd}, J_{1} \approx 8.3, J_{2}=2.4 \mathrm{~Hz}, 1 \mathrm{H}, \mathrm{Ar}-\mathrm{CH}\right), 7.42(\mathrm{~d}, J \approx 8.3 \mathrm{~Hz}, 1 \mathrm{H}, \mathrm{Ar}-\mathrm{CH})$, $7.04(\mathrm{~d}, J \approx 9.0 \mathrm{~Hz}, 2 \mathrm{Ar}-\mathrm{CH}), 6.87(\mathrm{~d}, J \approx 9.0 \mathrm{~Hz}, 2 \mathrm{H}, \mathrm{Ar}-\mathrm{CH}), 3.75,3.67$ (2dd, $J_{1} \approx 8.9$, $\left.J_{2} \approx 6.0 \mathrm{~Hz}, 2 \mathrm{H}, \mathrm{OCH}_{2}\right), 1.83-1.78(\mathrm{~m}, 1 \mathrm{H}, \mathrm{CH}), 1.24-1.18\left(\mathrm{~m}, 2 \mathrm{H}, \mathrm{CH}_{2}\right), 0.95(\mathrm{~d}, J \approx 6.7 \mathrm{~Hz}$, $\left.3 \mathrm{H}, \mathrm{CH}_{3}\right), 0.89\left(\mathrm{t}, J \approx 7.4 \mathrm{~Hz}, 3 \mathrm{H}, \mathrm{CH}_{3}\right) .{ }^{13} \mathrm{C} \mathrm{NMR}\left(125 \mathrm{MHz}, \mathrm{CDCl}_{3}\right): \delta(\mathrm{ppm})=163.48$ (COO), 158.11, 155.97, 142.27, 124.71 (Ar-C), 151.64, 139.19, 124.86, 122.15, 115.21 (Ar$C \mathrm{H}), 71.92\left(\mathrm{OCH}_{2}\right), 34.72(\mathrm{CH}), 26.13\left(\mathrm{CH}_{2}\right), 17.49,11.34\left(\mathrm{CH}_{3}\right)$. FT-IR: $\gamma\left(\mathrm{cm}^{-1}\right)=1728$ ( $\mathrm{C}=\mathrm{O}$ stretching), 1583-1504 (Ar-C $=\mathrm{C}$ stretching). $\mathbf{C}_{\mathbf{1 7}} \mathbf{H}_{\mathbf{1 8}} \mathbf{C I N O}_{\mathbf{3}}$ (319.79); Anal. Calc. (\%): C, 63.85; H, 5.67; N, 4.38. Found (\%): C, 64.12; H, 5.91; N, 4.17.

\section{4-((S)-3,7-Dimethyloctyloxy)phenyl 6-chloronicotinate (8b)}

Yield: $60 \%$; colorless crystals. ${ }^{1} \mathbf{H}$ NMR $\left(500 \mathrm{MHz} \mathrm{CDCl}_{3}\right): \delta(\mathrm{ppm})=9.08(\mathrm{~d}, J \approx 2.4 \mathrm{~Hz}$, $1 \mathrm{H}, \operatorname{Ar}-\mathrm{C} H), 8.31\left(\mathrm{dd}, J_{l} \approx 8.3, J_{2} \approx 2.4 \mathrm{~Hz}, 1 \mathrm{H} \mathrm{Ar}-\mathrm{C} H\right), 7.42(\mathrm{~d}, J \approx 8.3 \mathrm{~Hz}, 1 \mathrm{H}, \mathrm{Ar}-\mathrm{CH})$, $7.05(\mathrm{~d}, J \approx 9.1 \mathrm{~Hz} ; 2 \mathrm{H}, \mathrm{Ar}-\mathrm{CH}), 6.87(\mathrm{~d}, J \approx 9.1 \mathrm{~Hz}, 2 \mathrm{H}, \mathrm{Ar}-\mathrm{CH}), 3.93-3.89$ (m, 2H, OCH ${ }_{2}$, 1.79-1.70 (m, 1H, CH), 1.31-1.08 (m, 9H, CH, $\left.\mathrm{CH}_{2}\right), 0.88\left(\mathrm{~d}, J \approx 6.6 \mathrm{~Hz}, 3 \mathrm{H}, \mathrm{CH}_{3}\right), 0.80$ (d, $\left.J \approx 6.1 \mathrm{~Hz}, 6 \mathrm{H}, \mathrm{CH}_{3}\right) .{ }^{13} \mathrm{C} \mathbf{N M R}\left(125 \mathrm{MHz}, \mathrm{CDCl}_{3}\right): \delta(\mathrm{ppm})=163.45(\mathrm{COO})$, 157.25, 156.25, 143.59, $124.70(\mathrm{Ar}-\mathrm{C}), 151.64,140.05,124.39,122.16,115.21(\mathrm{Ar}-\mathrm{CH}), 66.78$ $\left(\mathrm{OCH}_{2}\right), 39.25,37.28,36.19,34.94\left(\mathrm{CH}_{2}\right), 29.84,27.99(\mathrm{CH}) 24.67,22.62,19.66\left(\mathrm{CH}_{3}\right)$. FT- 
IR: $\gamma\left(\mathrm{cm}^{-1}\right)=1713\left(\mathrm{C}=\mathrm{O}\right.$ stretching), 1586-1507 (Ar-C=C stretching). $\mathbf{C}_{\mathbf{2 2}} \mathbf{H}_{28} \mathbf{C l N O}_{3}$ (389.91); Anal. Calc. (\%): C, 67.77; H, 7.24; N, 3.59. Found (\%): C, 67.74; H, 7.27; N, 3.48.

\section{Results and Discussions}

\subsection{Liquid crystalline properties}

The mesomorphic properties of the pyridine based chiral compounds 4,7 and 8 were investigated by using optical polarizing microscope (POM) and differential scanning calorimeter (DSC). The phase transitions temperatures observed on heating and cooling cycles and corresponding transition enthalpies of the compounds are given in Table 1. The peak temperatures are given in degree Celsius and the numbers in parentheses indicate the transition enthalpy $(\Delta H)$. The mesomorphic behavior of all the compounds is summarized in Fig. 2.

Table 1 Mesophase, phase transition temperatures $\left({ }^{\circ} \mathrm{C}\right)$ and the corresponding transition enthalpies $(\Delta H \mathrm{~kJ} / \mathrm{mol})^{\mathrm{a}}$ of the compounds $\mathbf{4 a}, \mathbf{b} ; \mathbf{7 a}, \mathbf{b}$ and $\mathbf{8 a}, \mathbf{b}$.

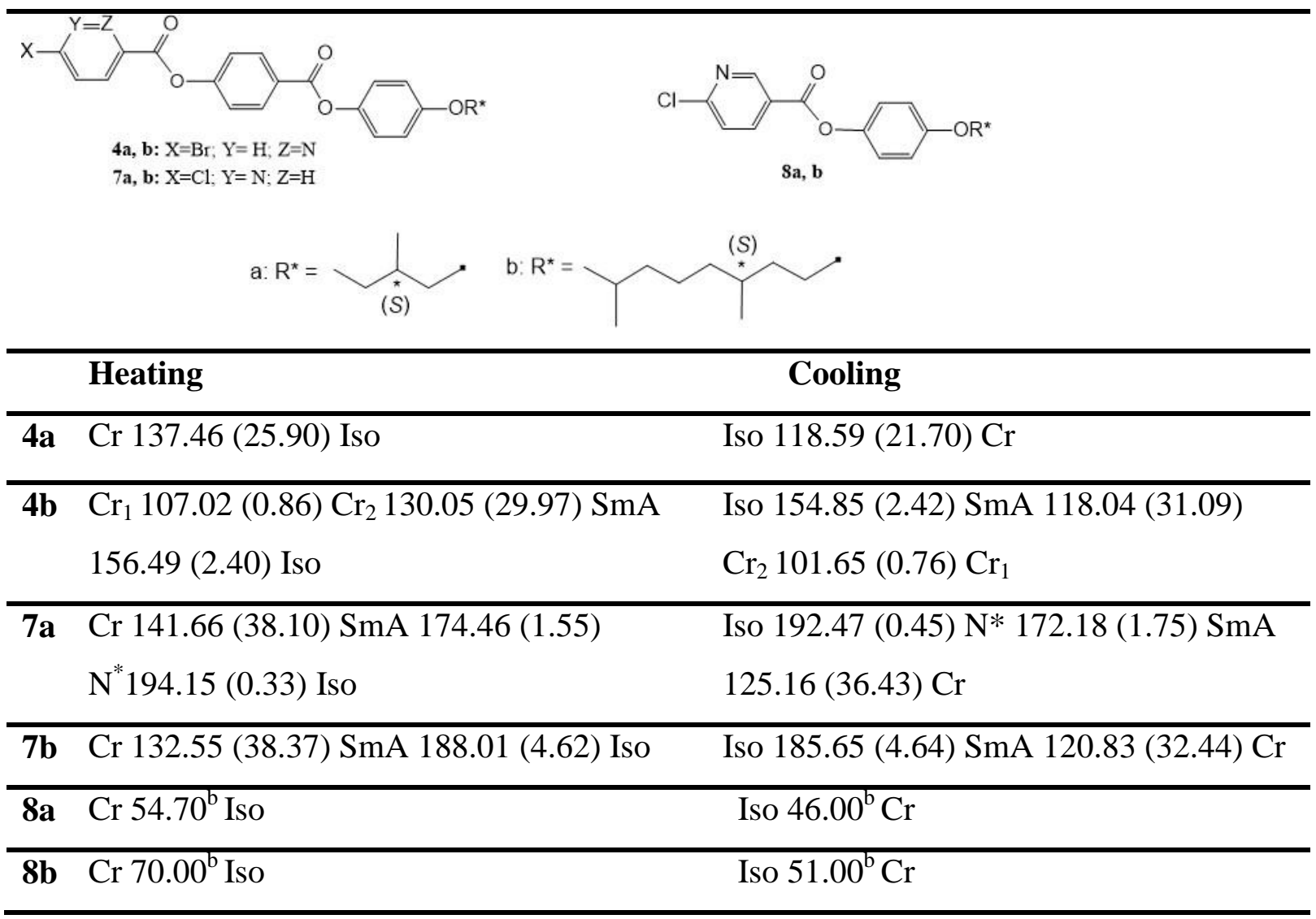


${ }^{\text {a }}$ Perkin-Elmer DSC-6; enthalpy values in italics in brackets taken from the $2^{\text {nd }}$ heating and cooling scans at a rate of $10{ }^{\circ} \mathrm{C} \cdot \mathrm{min}^{-1}$; Abbreviations: $\mathrm{Cr}=$ crystalline, $\mathrm{SmA}=$ smectic A mesophase, $\mathrm{N}^{*}=$ chiral nematic phase, Iso $=$ isotropic liquid phase. ${ }^{\mathrm{b}}$ These transitions were determined by PM.

On cooling, compound 4a composed of three aromatic rings substituted a bromo polar end group as well as $(S)$-2-methylbutoxy group shows only one reversible transition around 137 ${ }^{\circ} \mathrm{C}$, which corresponds to a crystal-to-isotropic phase transition by POM (see Fig.S27). On contrary, three transitions were detected in the DSC traces both during the heating and cooling runs for the compound $\mathbf{4 b}$ which is the analog substituted with $(S)$-3,7-dimethyloctyloxy chiral moiety of compound 4a. On heating, a phase transition sequence of crystal $\mathrm{Cr}_{1^{-}} \mathrm{Cr}_{2^{-}}$ SmA-Iso between $107{ }^{\circ} \mathrm{C}$ and $156{ }^{\circ} \mathrm{C}$ and the same reverse phase transition sequence with three exotherms were observed on cooling (Table 1 and Fig. 1). Depending on the presence of short branched end chain, compound $\mathbf{4 a}$ is non-mesogenic whereas compound $\mathbf{4 b}$ exhibits enantiotropic SmA mesophase appearing with an ordinary fan-shaped texture (see Fig. 2a).

Compounds 7a derived from 6-chloronicotinic acid substituted with (S)-2-methylbutoxy chiral moiety exhibits enantiotropic SmA phase as well as chiral nematic $\left(\mathrm{N}^{*}\right)$ phase. On cooling from isotropic phase, oily streaks texture of $\mathrm{N}^{*}$ phase firstly appeared at $192{ }^{\circ} \mathrm{C}$ and then homeotropic texture of SmA phase emerged at $172{ }^{\circ} \mathrm{C}$. It should be noted here that the enthalpy change during $\mathrm{N}^{*}$-Iso transition or vice versa transition is very small value. This is presumably a result from the short branched chain which increases the molecular biaxiality and so reduces the order change at the transition.[38] The homeotropic texture of SmA phase converted to the focal conic pattern after shearing the sample (see Fig. 2b).

However, compound $\mathbf{7 b}$ carrying longer and more branched chain by two methyl groups shows reversible transitions Cr-SmA-Iso which were detected in the DSC traces during the heating and the cooling runs. This result agrees with the work of Laschat et al.[39] who reported that homologous series of chloro- and bromo-terminated 5-phenylpyrimidine mesogens also form exclusively SmA phases and with the report of Goodby et al. that a chloro end-group acts as a strong SmA-promoter.[40,41] Additionally, compound 7b, which has a (S)-3,7-dimethyloctyloxy chiral moiety and a chloro polar head, shows richer mesomorphism and mesophase stability as compared with the mesomorphic behavior observed for pyridine-based calamitic esters carrying same chiral moiety.[42] On the other hand, it can be said that the use of a longer terminal alkyl chain in the members with three aromatic rings clearly allows to stabilize the formation SmA phase over larger temperature range in comparison of the introduction of shorter branched chain such as (S)-2-methylbutoxy 
316 group at the terminal. One point should be mentioned here that longer terminal chains reduce

317 the melting point by providing mobility and flexibility in the structure and this case allows 318 designing mesogens with desired properties for many applications.

319 Herein, we would also like to focus on the comparison between compounds 4a and 7a which 320 structurally differs by the nature of the halogen atom on the pyridine group and the position of 321 the nitrogen. It is known that the nature of halogen groups plays a significant role in the intra322 and inter-molecular interactions[43] which affect the packing of the molecules, that 323 predominantly influences mesophase stability. The main reason is not the strong polar 324 interactions at interfaces, as previously postulated in the literature.[44] Instead, the evidence 325 suggests that the effect is due to the electron-withdrawing effect of the chloro end-group, 326 which should reduce electrostatic repulsion between the alkoxy chains and increase attractive 327 van der Waals interactions between aromatic cores in the SmA phase.[45] Also, one point 328 should also be considered that the size and shape of the substituent which able to interact with 329 the mesogenic unit. Van der Waals volume of a substituent bounded to a phenyl ring shows a 330 linear dependence on phase transition.[46] In our case, the polar bromine of compound 4a 331 leads to a non-mesogenic behavior due to the lower electron-withdrawing effect of bromine 332 than that of chlorine as compared to compound $\mathbf{7 a}$ showing liquid crystallinity. 

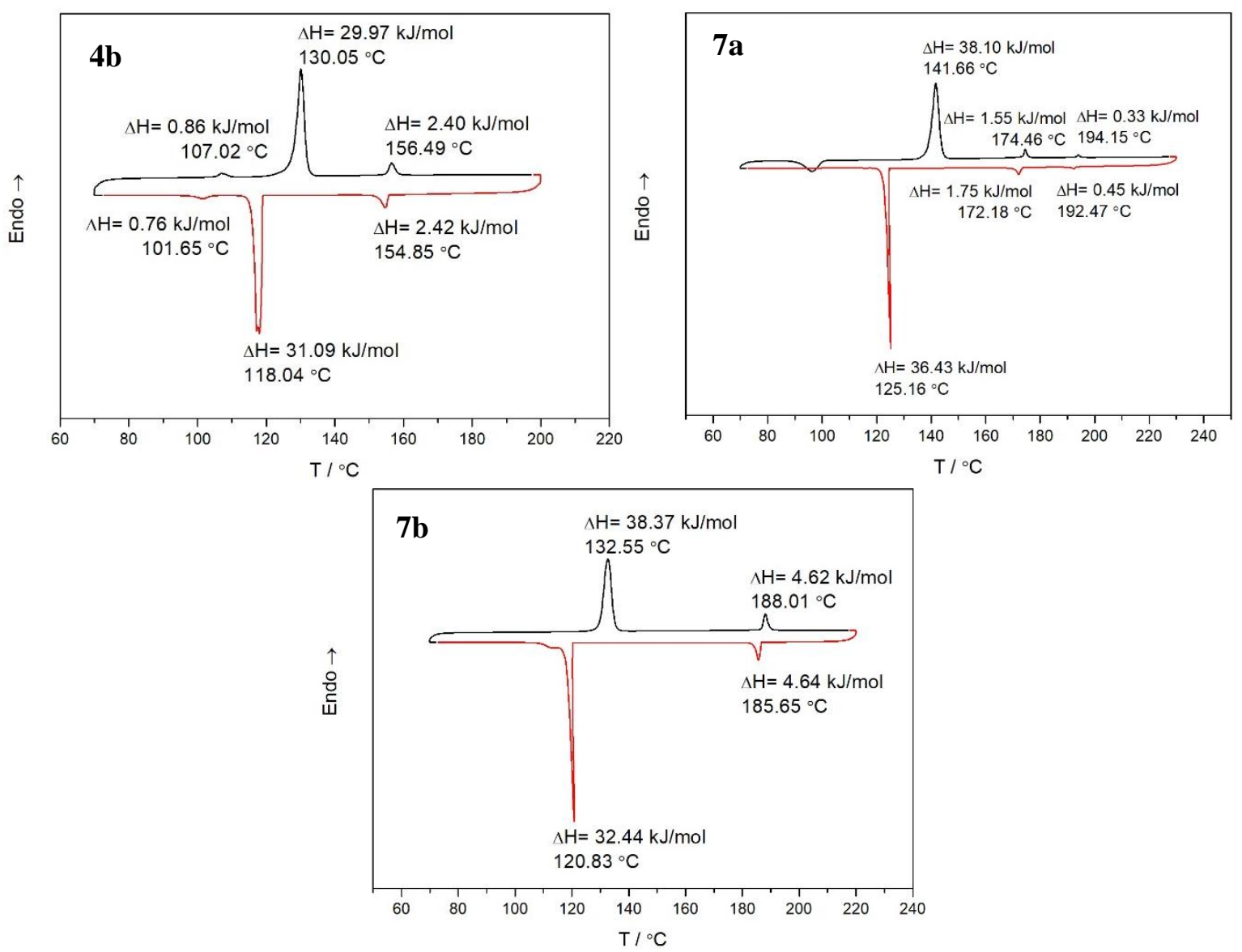

336 Fig. 1. DSC thermograms of compounds $\mathbf{4 b}$ and $\mathbf{7 a , b}$ on $2^{\text {nd }}$ heating and cooling $\left(10{ }^{\circ} \mathrm{C}\right.$ $337 \mathrm{~min}^{-1}$ ).
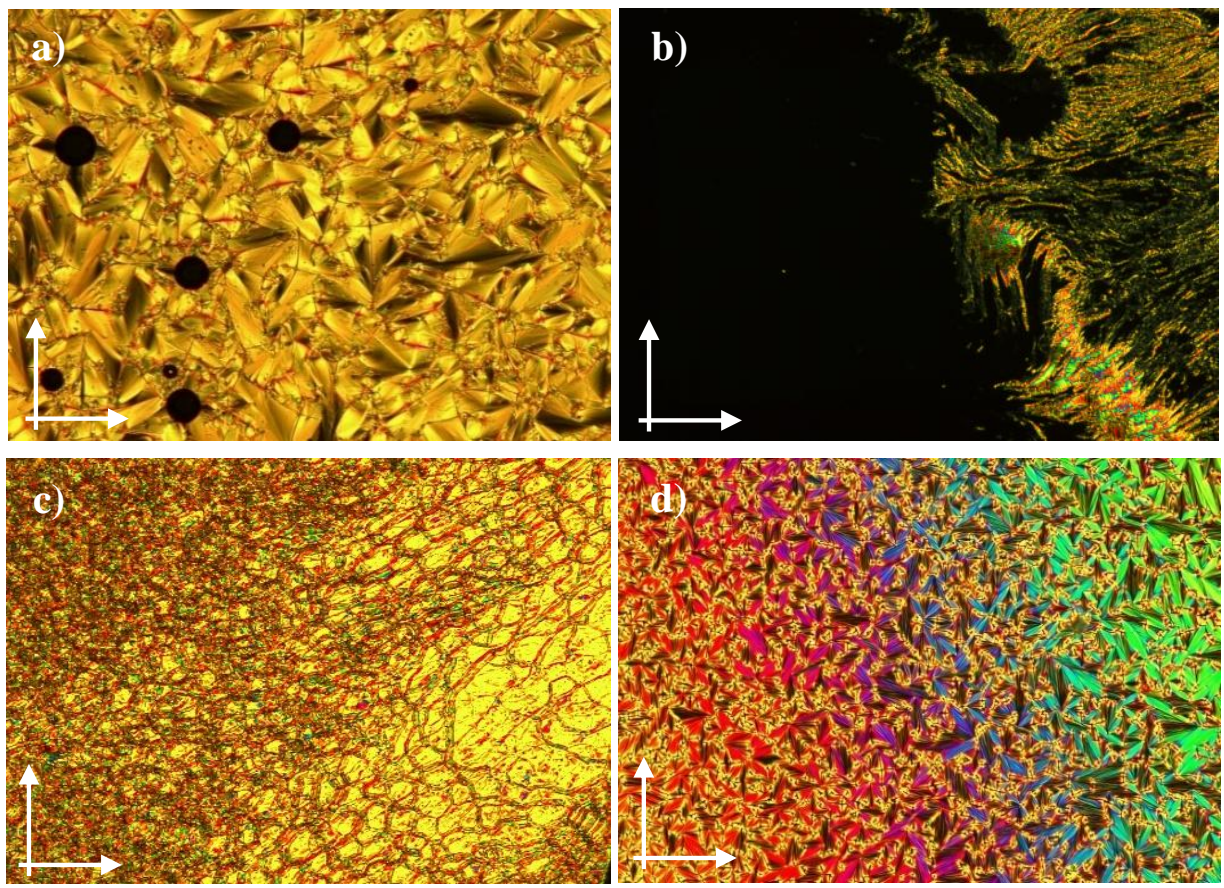

Fig. 2. Optical textures of mesophases of compounds $\mathbf{4 b}$ and $\mathbf{7 a , b}$ as observed between crossed polarizers (indicated by arrows) in ordinary glassplates on cooling (magnification 
$\times 100$ ); (a) fan-shaped texture of SmA mesophase of compound $\mathbf{4 b}$ obtained at $\mathrm{T}=151{ }^{\circ} \mathrm{C}$; (b) focal conic texture after shearing the homeotropic pattern of SmA mesophase of compound 7a obtained at $\mathrm{T}=148{ }^{\circ} \mathrm{C}$; (c) oily-streak texture of $\mathrm{N}^{*}$ phase of compound $7 \mathbf{a}$ obtained at $\mathrm{T}=$ $184{ }^{\circ} \mathrm{C}$; (d) fan-shaped texture of SmA mesophase of compound $7 \mathbf{b}$ obtained at $\mathrm{T}=160{ }^{\circ} \mathrm{C}$.

Compounds $\mathbf{8 a}$ and $\mathbf{8 b}$ show reversible crystal-isotropic transition that corresponds to a nonmesogenic behavior (see Table 1 and Fig.S28). This behavior indicates that only two aromatic rings are not sufficient to obtain liquid crystallinity. Surprisingly, melting point of compound $\mathbf{8 b}$ with more branched and longer chain $\sim 15{ }^{\circ} \mathrm{C}$ higher than that of compound $\mathbf{8 a}$.

\subsection{SAXS Measurements}

The mesophases of compounds $\mathbf{7 a}$ and $\mathbf{7 b}$ derived from 6-chloronicotinic acid were confirmed by XRD analysis. The SAXS patterns recorded on $\mathbf{7 b}$ compound confirm the presence of two reversible phase transitions around 130 and $180^{\circ} \mathrm{C}$. The SAXS pattern of the high temperature phase is typical of an isotropic state with a broad halo in the small angles region $(34.4 \AA)$ and a broad halo in the wide angles region $(4.9 \AA)$, arising from the mean distances between the long and the short molecular axes, respectively, in a disordered state (Fig. 3a and 3b). Below $130{ }^{\circ} \mathrm{C}$, the SAXS patterns are typical of a crystalline phase with many sharp diffraction peaks in the small and wide angles regions (Fig. 3c). On contrary, the SAXS patterns recorded between 130 and $180{ }^{\circ} \mathrm{C}$ are characterized by the presence of two sharp reflections in the small angles region and a broad halo in the wide-angle region. Based on the POM observations, showing the formation of SmA phase, the reflections observed at 33.4 and $16.7 \AA$ in the small angle region were indexed as the (001) and (002) reflections of a lamellar phase. In the wide-angle region, the broad halo centered at $4.75 \AA$ was associated to the mean distance between the alkyl chains in a molten state and confirms the liquid crystalline nature of the phase. The size of the molecule is a fully extended state was estimated to $27.5 \AA$ using a Chem3D molecular model. The lamellar period $\mathrm{d}=33.4 \AA$ is only slightly larger than the molecular length (Fig. 3d). Thus, the smectic phase is constituted of monolayers of molecules slightly shifted along the director. 
389

390

391

392

393

394

395

396

397

398

399

400

401

402

403

404

405

406

407

408

409

410

411

412

c)

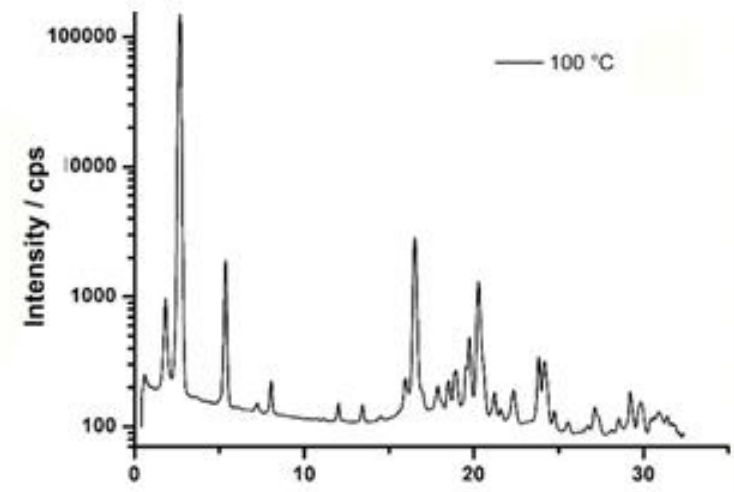

$20 I^{\circ}$ b)

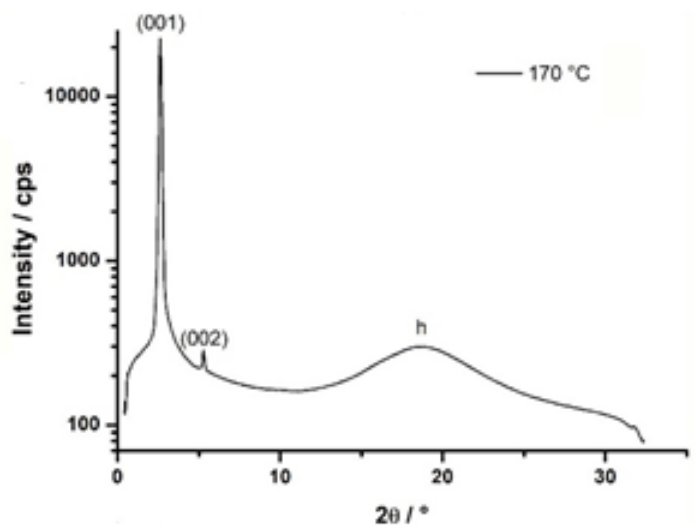

d)

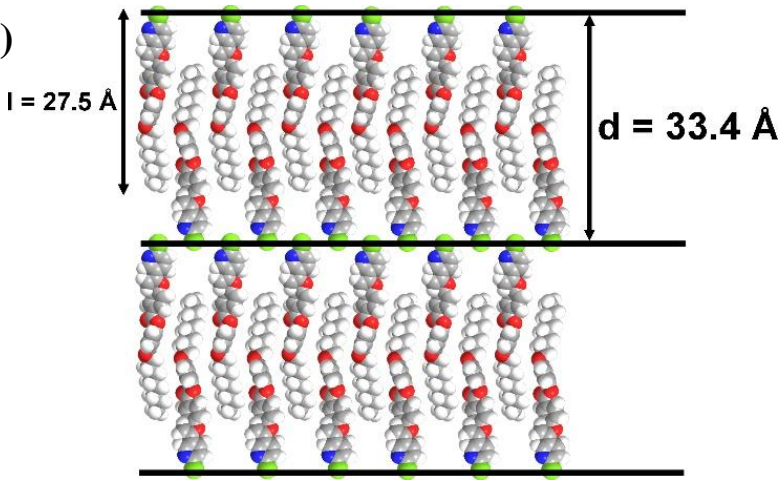

Fig. 3. SAXS pattern of $\mathbf{7 b}$ compound recorded at different temperatures (a) $\mathrm{T}=200{ }^{\circ} \mathrm{C}$; (b) $\mathrm{T}=170 \mathrm{C}^{\circ}$; (c) $\mathrm{T}=200{ }^{\circ} \mathrm{C}\left(2^{\text {rd }}\right.$ heating $)$ and $\left.\mathrm{d}\right)$ Proposed organization for $7 \mathbf{b}$ in the SmA mesophase at $170{ }^{\circ} \mathrm{C}$ (extended length $\sim 27.5 \AA$ (Dreiding model); atom: white = hydrogen, grey $=$ carbon, blue $=$ nitrogen, green $=$ chloride, red $=$ oxygen .

From the XRD data, an organization model can be proposed and his presented in Fig. 3d. The lamellar distance moderately increases by less than $1 \%$ between 140 and $170{ }^{\circ} \mathrm{C}$ and a layer 413

414

415 
422

423

424

425

426

427

428

429

430

431

432

433

434

435

436

437

438

439

440

441

442

443

444

445

446

447

448

449

450

451

452 a)

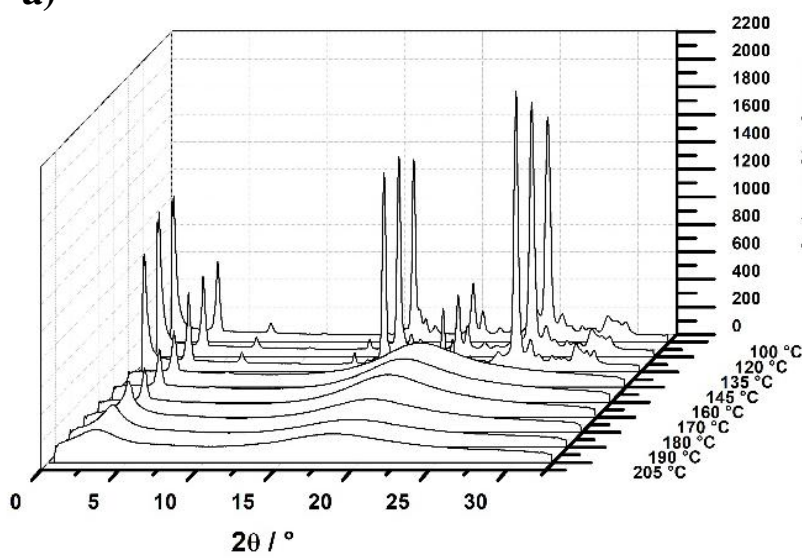

b)

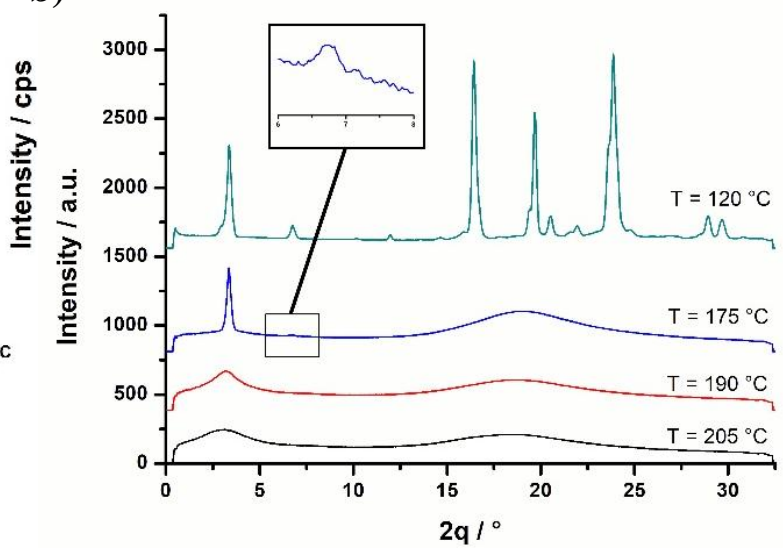

Fig. 4. (a) SAXS patterns recorded on 7a compound upon heating (b) Selected temperaturedependent SAXS patterns recorded on 7a compound upon cooling (insert: zoom of the area 6$8^{\circ}$ in $2 \theta$ of the curve at $175^{\circ} \mathrm{C}$ ).

The SAXS patterns of compound 7a measured at $205{ }^{\circ} \mathrm{C}$ is characteristic of an isotropic state with one broad peak in the small angles region centered at $28.5 \AA$ and another one in the wide angles region at $4.8{ }^{\circ} \mathrm{C}$. Upon cooling, a clear narrowing of the diffraction peak in the small angles region is observed around $195^{\circ} \mathrm{C}$ and is in line with the formation of a nematic phase with a subtle ordering of the rod-like molecules (see Fig. 4). By POM observations, this phase was assigned as a chiral nematic phase $\left(\mathrm{N}^{*}\right)$. Upon further cooling, another phase transition is clearly detected between 185 and $175^{\circ} \mathrm{C}$. The first order reflection centered at $26.3 \AA$ at 175 ${ }^{\circ} \mathrm{C}$ became really sharp and a second order reflections appeared at $13.2 \AA$. These two reflections, based on the POM observations, can be indexed as the first two (001) and (002) reflections of a lamellar phase. The broad halo centered at associated to the carbon chains in a molted state, confirms that the compound is in a liquid crystalline state. The compound remains in this smectic phase down to $140^{\circ} \mathrm{C}$ and a slight decrease of the interlayers distance is observed with a layer thermal contraction coefficient of $-0.01 \AA . \mathrm{K}^{-1}$. The interlayers distance is comparable to the length of the molecule in a fully extended conformation $1=24.5$ $\AA \sim \mathrm{d}$, meaning that the SmA phase is formed of monolayers of molecules arranged in slightly shifted head-to-tail fashion like $7 \mathbf{b}$ compound (See model Fig. 3d). At $120{ }^{\circ} \mathrm{C}$, the compound is clearly in a crystalline state and several sharp diffraction peaks are observed on the whole $2 \theta$ range. On heating, the reverse phase transition sequence is observed and the compounds transit from a crystalline phase to the SmA phase at $135-145^{\circ} \mathrm{C}$, then from the SmA phase to the $\mathrm{N}^{*}$ phase at $170-180{ }^{\circ} \mathrm{C}$ and finally from the $\mathrm{N}^{*}$ phase to the isotropic phase at $195-205$ 
${ }^{\circ} \mathrm{C}$. Thus, all the transitions are perfectly reversible and the phase transition temperatures detected by SAXS are fully consistent with the temperature measured by DSC.

\section{Conclusion}

In this article, we have synthesized and characterized new pyridine based rod-like compounds composed of a chloro- or bromo-pyridine polar head linked to one or two benzene rings through ester linkers and a flexible a $(S)$-2-methylbutoxy or (S)-3,7-dimethyloctyloxy chiral end chain on the other side. The chain length and the type of the halogen atom have been varied to understand the relationship between the structure and mesogenic properties. The chlorine substituted two aromatic rings derivatives exhibit non-mesogenic behavior, whereas members with three aromatic rings carrying a (S)-3,7-dimethyloctyloxy or (S)-2methylbutoxy group exhibit SmA phase. A chiral nematic phase $\left(\mathrm{N}^{*}\right)$ was also observed for the derivative with a $(S)$-2-methylbutoxy terminal chain. Additionally, the replacement of chlorine with bromine atom as well as the position of nitrogen atom in the pyridine ring lead to disappearing of liquid crystallinity for derivative carrying the same chiral moiety. However, it can be said that the mesophase stability decreases for bromine substituted analog terminated with $(S)$-3,7-dimethyloctyloxy group. The mesophases of compounds derived from 6-chloronicotinic acid were confirmed by XRD measurement. The organization in the SmA mesophase of (S)-3,7-dimethyloctyloxy substituted analog is constituted of monolayers of molecules slightly shifted along the director. What's emerges from our work is that the occurence of liquid crystalline phase strongly depends on the nature of the halogen atoms and the introduction of the chlorine atom into the structure due to its more electron-withdrawing effect is more favorable.

\section{Acknowledgement}

This research has been supported by The Scientific and Technological Research Council of Turkey (TÜBITAK), Turkey with the Project Number 116Z465. The authors are grateful to the TÜBİTAK-2232 programme for a support with Project Number 118C273.

\section{Appendix A. Supplementary data}

Electronic supplementary information (ESI) is available. 
${ }^{1}$ Tschierske C. Development of Structural Complexity by Liquid Crystal Self-assembly. Angew. Chem. Int. Ed. 2013; 52: 8828-8878.

${ }^{2}$ Kohout M, Bubnov A, Šturala J, Novotná V, Svoboda J. Effect of alkyl chain length in the terminal ester group on mesomorphic properties of new chiral lactic acid derivatives. Liq. Cryst. 2016; 43: 1472-1475.

${ }^{3}$ Lehmann M, Kestemont G, Aspe RG, Buess-Herman C, Koch MHJ, Debije MG, Piris J, De Haas MP Warman JM, Watson MD, Lemaur V, Cornil J, Geerts YH, Gearba R, Ivanov DA. High Charge-Carrier Mobility in $\pi$-Deficient Discotic Mesogens: Design and StructureProperty Relationship. Chem.-Eur. J. 2005; 11: 3349-3362.

${ }^{4}$ O'Neill M, Kelly SM. Liquid Crystals for Charge Transport, Luminescence, and Photonics. Adv. Mater. 2003; 15: 1135-1146.

${ }^{5}$ Castillo-Vallés M, Martínez-Bueno A, Giménez R, Sierra T, Blanca Ros M. Beyond liquid crystals: new research trends for mesogenic molecules in liquids. J. Mater. Chem. C 2019; 7:14454-14470.

${ }^{6}$ Demus D, Goodby J, Gray GW, Spiess H-W, Vill V. Handbook of liquid crystals vol. IIA, Weinheim (1998), Chapter VI p 133-187.

${ }^{7}$ Lagerwall JPF, Scalia G. A new era for liquid crystal research: Applications of liquid crystals in soft matter nano-, bio- and microtechnology. Curr. Appl. Phys. 2012; 12: 13871412.

${ }^{8}$ Goodby JW. Nano-objects-sculpting and shape in molecular material design (The Pierre Gilles de Gennes ILCS prize lecture). Liq. Cryst. 2019; 46: 1901-24.

${ }^{9}$ Girotto CE, Bechtold IH, Gallardo H. New liquid crystals derived from thiophene connected to the 1,2,4-oxadiazole heterocycle. Liq. Cryst. 2016; 12: 1768-1777.

${ }^{10}$ Ghosh T, Lehmann M. Recent Advances in Heterocycle-Based Metal-Free Calamitics. J. Mater. Chem. C. 2017; 5: 12308-12337.

11 Lagerwall JPF, Giesselmann F. Current Topics in Smectic Liquid Crystal Research. ChemPhysChem. 2006; 7: 20-45.

${ }^{12}$ Keith C, Reddy RA, Tschierske C. The first example of a liquid crystalline side-chain polymer with bent-core mesogenic units: ferroelectric switching and spontaneous achiral symmetry breaking in an achiral polymer. Chem. Commun. 2005; 7: 871-873. 
${ }^{13}$ Ocak H, Bilgin-Eran B, Tschierske C, Baumeister U, Pelzl G. Effect of fluorocarbon chains on the mesomorphic properties of chiral imines and their complexes with copper(II). J. Mater. Chem. 2009; 19: 6995-7001.

${ }^{14}$ Collings PJ, Hird M. Introduction to Liquid Crystals. Taylor and Francis: London. 2001.

${ }^{15}$ Ocak H, Bilgin-Eran B, Prehm M, Schymura S, Lagerwall JPF, Tschierske C. Effects of chain branching and chirality on liquid crystalline phases of bent-core molecules: blue phases, de Vries transitions and switching of diastereomeric states. Soft Matter. 2011; 7: 8266-8280.

16 Clark NA, Lagerwall ST. Submicrosecond bistable electro-optic switching in liquid crystals. Appl. Phys. Lett. 1980; 36: 899-901.

${ }^{17}$ Bubnov A, Novotná V, Hamplová V, Kašpar M, Glogarová M. Effect of multilactate chiral part of liquid crystalline molecule on mesomorphic behaviour. J. Mol. Struct. 2008; 892: 151157.

${ }^{18}$ Das B, Pramanik A, Kumar Das M, Bubnov A, Hamplová V, Kašpar M. Mesomorphic and structural properties of liquid crystal possessing a chiral lactate unit. J. Mol. Struct. 2012; 1013: 119-125.

${ }^{19}$ Kohout M, Bubnov A, Šturala J, Novotná V, Svoboda J. Effect of alkyl chain length in the terminal ester group on mesomorphic properties of new chiral lactic acid derivatives. Liq. Cryst. 2016; 43: 1472-1485.

${ }^{20}$ Ocak H, Poppe M, Bilgin-Eran B, Karanlık G, Prehm M, Tschierske C. Effects of molecular chirality on self-assembly and switching in liquid crystals at the cross-over between rod-like and bent shapes. Soft Matter. 2016; 12: 7405-7422.

${ }^{21}$ Tschierske C. Mirror symmetry breaking in liquids and liquid crystals. Liquid Crystals. 2018; 45: 2221-52.

${ }^{22}$ Abberley JP, Killah R, Walker R, Storey JMD, Imrie CT, Salamończyk M, Zhu C, Gorecka E, Pociecha D. Heliconical smectic phases formed by achiral molecules. Nature Communications. 2018;9:228.

${ }^{23}$ Salamonczyk M, Vaupotič N, Pociecha D, Walker R, Storey JMD, Imrie CT, Wang C, Zhu C, Gorecka E. Multi-level chirality in liquid crystals formed by achiral molecules. Nature Communications. 2019;10: 1922.

${ }^{24}$ Paterson DA, Abberley JP, Harrison WTA, Storey JMD, Imrie CT. Cyanobiphenyl-based liquid crystal dimers and the twist-bend nematic phase. Liquid Crystals. 2017; 44: 127-46.

${ }^{25}$ Reddy RA, Tschierske C. Bent-core liquid crystals: polar order, superstructural chirality and spontaneous desymmetrisation in soft matter systems. J. Mater. Chem. 2006; 16: 907-961. 
${ }^{26}$ Ong LK, Ha ST, Yeap GY, Lin HC. Heterocyclic pyridine-based liquid crystals: synthesis and mesomorphic properties. Liq. Cryst. 2018; 45: 1574-1584.

${ }^{27}$ Campbell NL, Duffy WL, Thomas GI, Wild JH, Kelly SM, Bartle K, O'Neill M, Minter V, Tuffin RP. Nematic 2,5-disubstituted thiophenes. J. Mater. Chem. 2002; 12: 2706-2721.

${ }^{28}$ Burrow MP, Gray GW, Lacey D, Toyne KJ. The synthesis and liquid crystal properties of some 2,5-disubstituted pyridines. Liq. Cryst. 1988; 3: 1643-1653.

${ }^{29}$ Chia WL, Tsai CY. Synthesis and mesomorphic properties of a series of phenyl 6-(4alkoxyphenyl) nicotinates. Heterocycles. 2011; 83: 1057-1065.

${ }^{30}$ Chia WL, Lin CW. Synthesis and thermotropic studies of a novel series of nematogenic liquid crystals 2-(6-alkoxynaphthalen-2-yl)-5-cyanopyridines. Liq. Cryst. 2013; 40: 922-931.

${ }^{31}$ Petrov VF. Nitrogen-containing fused heterocycles as the structural fragments in calamitic liquid crystals. Liq. Cryst. 2001; 28: 217-240.

${ }^{32}$ Nash JA, Gray GW. Studies of Some Heterocyclic Mesogens. Mol. Cryst. Liq. Cryst. 1974; 25: 299-321.

${ }^{33}$ Guzeller D, Ocak H, Bilgin-Eran B, Prehm M, Tschierske C. Development of tilt, biaxiality and polar order in bent-core liquid crystals derived from 4'- hydroxybiphenyl-3-carboxylic acid. J. Mater. Chem. C 2015; 3: 4269-4282.

34 Jocelyn PC, Polgar N. Methyl-substituted $\alpha \beta$-unsaturated acids. Part I. J. Chem. Soc. 1953; 132-137.

${ }^{35}$ Kondo H, Okazaki T, Endo N, Mihashi S, Yamaguchi A, Tsuruta H, Akutagawa S. Jpn. Kokai Tokkyo Koho 1988; JP 63033351 A 19880213.

${ }^{36}$ Chin E, Goodby JW. A Protection-Deprotection Method for the Synthesis of Substituted Benzoyloxybenzoates. Mol. Cryst. Liq. Cryst.1986; 141: 311-320.

${ }^{37}$ Kuo SC, Lee KH, Huang LJ, Chou LC, Wu TS, Way TD, Chung JG, Yang JS, Huang CH, Tsai MT. PCT Int. Appl. 2012; WO 2012009519 A1 20120119.

${ }^{38}$ Chan TN, Lu Z, Yam W-S, Yeap G-Y, Imrie CT. Non-symmetric liquid crystal dimers containing an isoflavone moiety. Liq. Cryst.. 2012; 39: 393-402.

${ }^{39}$ Starkulla GF, Kapatsina E, Baro A, Giesselmann F, Tussetschlager S, Kaller M, Laschat S. Influence of spacer chain lengths and polar terminal groups on the mesomorphic properties of tethered 5-phenylpyrimidines. Beilstein J. Org. Chem. 2009; 5: No.63.

${ }^{40}$ Cowling SJ, Hall AW, Goodby JW. Effect of terminal functional group size on ferroelectric and antiferroelectric properties of liquid crystals. Liq. Cryst. 2005; 32: 1483-1498. 
${ }^{41}$ Cowling SJ, Goodby JW. Interfacial layer interactions: their effects on synclinic and anticlinic smectic mesophase behaviour in liquid crystals. Chem. Commun. 2006; 39: 4107-4109.

${ }^{42}$ Karanlık G, Ocak H, Bilgin Eran B. New pyridine based liquid crystalline esters with different terminal chains. J. Mol. Struct. 2019; 1198: 126930-126938.

${ }^{43}$ Petrov VF. Halogenation in Achiral Calamitic Liquid Crystals. II Terminal and Linking Substitutions. Mol. Cryst. Liq. Cryst. 2010; 517: 27-42.

${ }^{44}$ Goodby JW, Saez IM, Cowling SJ, Görtz V, Draper M, Hall AW, Sia S, Cosquer G, Lee SE, Raynes EP. Transmission and Amplification of Information and Properties in Nanostructured Liquid Crystals. Angew. Chem. Int. Ed. 2008; 47: 2754-2787.

45 Rupar I, Mulligan KM, Roberts JC, Nonnenmacher D, Giesselmann F, Lemieux RP. Elucidating the smectic A-promoting effect of halogen end-groups in calamitic liquid crystals. J. Mater. Chem. C. 2013; 1: 3729-3735.

${ }^{46}$ Yeap GY, Osman F, Imrie CT. Non-symmetric chiral liquid crystal dimers. Preparation and characterisation of the (S)-(benzylidene-4'-substitutedaniline)-2"-methylbutyl-4"'-(4"'-phenyloxy)-benzoateoxy)hexanoates. J Mol Struct. 2016; 1111: 118-25. 